\title{
NUPR1 Promotes the Proliferation and Migration of Breast Cancer Cells by Activating TFE3 Transcription to Induce Autophagy
}

\section{Heng Xiao}

The Affiliated Zhuzhou Hospital of XiangYa School of Medicine CSU

Jing Long

The Affiliated Zhuzhou Hospital Xiangya Medical College CSU

\section{Xiang Chen}

Laboratory Medicine Centre of zhuzhou central hospital

Mi-Duo Tan ( $\square$ mmdduuttaa987@163.com )

The Affiliated Zhuzhou Hospital of Xiangya Medical College CSU

\section{Research Article}

Keywords: breast cancer, NUPR1, TFE3, autophagy

Posted Date: December 1st, 2021

DOI: https://doi.org/10.21203/rs.3.rs-1126403/v1

License: (a) (i) This work is licensed under a Creative Commons Attribution 4.0 International License. Read Full License 


\section{Abstract}

Background: Breast cancer is a commonplace carcinoma in females. Recurrence and metastasis are the main problems affecting the survival rate of patients. The fundamental reason is the lack of understanding of the mechanism of breast cancer metastasis. This study aims to deliberate on the efficaciousness of Nuclear protein 1 (NUPR1)-mediated autophagy on breast cancer metastasis.

Methods: The proliferation, migration and invasion ability of breast cancer cells were appraised by CCK-8, wound healing, and colony formation, as well as transwell assay. The relationship between NUPR1 and Translocation factor E3 (TFE3) was appraised by qPCR, western blot and ChIP. Migration-invasion-related proteins and autophagy-related proteins were appraised by western blot. The effects of NUPR1 on malignancy formation and metastasis were studied in vivo.

Results: NUPR1 was upregulated in breast cancer cells and tissues. NUPR1 knockdown restrained the proliferation, migration and invasion of ZR-75-30 cells. Moreover, NUPR1 knockdown restrained malignancy formation and metastasis in vivo. Mechanically, NUPR1 promoted autophagy through activation of TFE3 transcription, thereby regulating the process of breast cancer metastasis.

Conclusion: This paper elucidates the molecular mechanism of NUPR1 promoting breast cancer metastasis by activating autophagy through TFE3 signaling pathway, which provided biological basis for intervention of blocking distant metastasis.

\section{Background}

Breast cancer is the most commonplace carcinoma among women in the world, with the highest prevalence among women's cancers(1). Despite the continuous development and improvement of clinical surgical treatment, radiotherapy, chemotherapy, biological therapy, targeted therapy, and other technologies have made the subsistence rate of invalids with breast cancer greatly ameliorated, the recurrence and metastasis are still the main problems affecting the survival rate of patients(2). Tumor invasion and metastasis is a core molecular biological event in the occurrence and development of malignant tumors, and it is also a key factor affecting the prognostic of breast cancer sufferers(3). Contemporarily, there is still a lack of effective means in clinical treatment of breast cancer metastasis. The fundamental reason is the deficiency of comprehensive awareness of the mechanism of breast cancer metastasis. Correspondingly, it is of appreciable meaningfulness to study the regulatory mechanism of cell proliferation, invasion and metastasis of breast cancer.

Autophagy is an important biological process for maintaining cell homeostasis, which promotes intracellular homeostasis by degrading a variety of targets, such as proteins, organelles and microorganisms $(4,5)$. In recent years, the role of autophagy in regulating tumor invasion and metastasis has become a research hotspot $(6,7)$. Autophagy-related genes are found to be associated with breast cancer based on gene expression profiles(8). The overexpression of LC3 and ATG17, the autophagyassociated proteins, in breast cancer tissues is significantly correlated with the high metastasis rate of 
patients(9). Moreover, the overexpression of ATG10, the key protein of autophagy vesicle formation, in breast cancer tissue suggests that the patients have poor prognosis and the possibility of lymph node metastasis(10). Beclin1 is a key regulator of autophagy signaling pathway, which can regulate the cycle of breast cancer cells and promote the invasion and metastasis of breast cancer cells. Beclin1 can be regarded as one of the key oncogenes of breast cancer(11). Our previous research also found that the index of autophagy in breast cancer tissue increased conspicuously, and inhibition of autophagy was conducive to the therapy of breast cancer(12). Nevertheless, the mechanism of autophagy activation in breast cancer cells is currently controversial. Exploring the mechanism of autophagy activation in breast cancer has positive significance for the intervention and treatment of invasion and metastasis of breast cancer.

Nuclear protein 1 (NUPR1) is a transcriptional regulator located in the nucleus, which can be induced under different stress environments and conditions, and participate in various physiological processes, exempli gratia, cell proliferation, apoptosis, cell cycle regulation, tumor cell invasion and metastasis(1315). NUPR 1 is the key molecule to control autophagy. NUPR1 participates in the regulation of autophagy and affects tumor invasion and metastasis. Various researches reported that NUPR1 can initiate the transcription of autophagy-lysosomal pathway related genes under stress environment, thus initiating the expression of a series of genes, covering almost the whole process of regulation(16). A large number of researches have shown that there is overexpression of NUPR1 in breast cancer, which is considered to be an essential factor in the deficient prognosis of patients. Besides, NUPR1 can provoke the proliferation of human breast cancer cells in the inoculated organs, which is related to its metastasis(17-19).

Translocation factor E3 (TFE3) can regulate the development of autophagy by regulating the expression of genes related to autophagy and lysosome, and promote the fusion of autophagosome and lysosome, which plays an important role in protein degradation(20). Furthermore, inhibiting TFE3 can inhibit the level of autophagy and weaken the capacity of invasion and metastasis of carcinoma(21). TFE3 overexpresses in breast cancer can contribute to the activation of autophagy(12).

In conclusion, we speculate that NUPR1 activates TFE3 transcription to induce autophagy activation, thereby enhancing the invasion ability and promoting distant metastasis. This study aims to deliberate on the efficaciousness of NUPR1-mediated autophagy on carcinoma metastasis, and to provide theoretical and experimental basis for the research of new treatments for breast cancer.

\section{Materials And Methods}

\section{Clinical samples}

The subjects were 30 breast cancer tissue specimens and matched paracancerous tissue specimens, all of which were confirmed by the case and did not receive chemotherapy or radiotherapy. There were 19 invalids without lymph node metastasis and 11 invalids with lymph node metastasis. All specimens were collected on the morning of the operation. The specimens were instantly soaked in liquid nitrogen and hoarded at $-80^{\circ} \mathrm{C}$ until subsequent study. All human participants provided written informed consent to 
participate in this research, which was approved by Ethics Committee of The Affiliated Zhuzhou Hospital of Xiangya Medical College CSU.

\section{Cell culture}

MCF-7, MDA-MB-231, ZR-75-30, and T47D, as well as normal breast epithelial cell MCF-10A were cultured in Dulbecco's modified eagle medium (DMEM), RPMI-1640 or Leibovitz's Lmur 15 medium containing $10 \%$ fetal bovine serum (FBS), $1 \%$ streptomycin and penicillin, respectively. All of cells were propagated at $37^{\circ} \mathrm{C}$ with $5 \% \mathrm{CO}_{2}$.

\section{Cell transfection}

The construction of lentiviral vectors of shRNA (sh)-NUPR1, sh-TFE3 and sh-ATG5, as well as sh-NC, lentivirus packaging and titer determination were completed by GenePharma Co., Ltd (Shanghai, China). ZR-75-30 cells in logarithmic phase were collected and seeded into 24 -well plates $\left(5 \times 10^{4}\right.$ cells/well). The experimental cells were divided into sh-NC and corresponding transfection groups. The sh-NC and the corresponding sh-NUPR1, sh-TFE3 or sh-ATG5 lentivirus infected cells respectively, and the culture medium was changed after $12 \mathrm{~h}$ of culture. $0.5 \mathrm{mg} / \mathrm{L}$ puromycin solution was used for screening after 72 $\mathrm{h}$ of transfection, and the fluorescence expression was observed under fluorescence microscope. Stable transfection cell lines were obtained after 2 weeks of screening.

\section{Immunohistochemistry (IHC)}

The tissue specimens were immovable with $10 \%$ formaldehyde, embedded in paraffin, sliced up $4 \mu \mathrm{m}$ slices, baked in $60^{\circ} \mathrm{C}$ oven for $30 \mathrm{~min}$, dewaxed with xylene for 3 times, 5 min each time, and rehydrated with decreasing gradient ethanol. After rinsed with phosphate buffer solution, the sections were placed in citrate buffer solution at high temperature to repair the exposed antigen, then rinsed with Phosphate buffered saline (PBS). The slices were incubated in $3 \% \mathrm{H}_{2} \mathrm{O}_{2}$ at constant temperature for 10 min to interdict endogenous peroxidase, and rinsed with distilled water and PBS. The protein were sealed and placed at ambient temperature for $10 \mathrm{~min}$. Sheep anti-human primary antibody was added respectively, and the slices were placed in the $4^{\circ} \mathrm{C}$ refrigerator overnight. The next day, the slices were rinsed with distilled water and PBS. After that rabbit anti-sheep secondary antibody labeled with horseradish peroxidase (HRP) was added to the slices, and the slices were incubated at $37^{\circ} \mathrm{C}$ for $30 \mathrm{~min}$. After rinsed with distilled water and PBS, the slices were stained with diaminobenzidine kit for 2 5 min. After the completion of color development, the slices were rinsed with distilled water to stop color development, and then re-stained with hematoxyl for 3 5 min. The slices were differentiated with $1 \%$ hydrochloric acid and alcohol for $3 \sim 5 \mathrm{~s}$. After fully rinsed, the slices were dehydrated by gradient ethanol incrementing, and the slices were roasted and sealed with neutral gum.

\section{Cell counting kit-8 (CCK-8) assay}


ZR-75-30 cells were seeded into the culture plate with 5000 cells per well. After 24,48 and $72 \mathrm{hrs}$ of culture respectively, appropriate amount of CCK-8 solution was blended and nurtured at $37^{\circ} \mathrm{C}$ with $5 \%$ $\mathrm{CO}_{2}$ for $4 \mathrm{hrs}$. The OD value was evaluated by automatic enzyme labeling instrument after shaking for 1 $\min$.

\section{Colony formation}

The logarithmic growth phase cells were seeded into the $6 \mathrm{~cm}$ petri dish with 1000 cells per well. Threemultiple pores were set up, and the cell state was observed every 3 days. After 7 days of culture, the cell colonies were sluiced with PBS twice, fastened with $4 \%$ paraformaldehyde, pigmented with Giemsa (Sigma, USA), and then rinsed with $\mathrm{ddH}_{2} \mathrm{O}$ for 2 times. The number of cell clones was observed under microscope.

\section{Flow cytometry}

After 5 days of lentivirus infection, the cells were prepared into cell suspension, and three-multiple pores were set up in the each group. Centrifuge and discard the supernatant, cell suspension with a cell density of $1 \times 10^{5} / \mathrm{mL}$ was collected, sluiced with PBS, and incubated with Annexin V-FITC (ANNEX300F, Bio-Rad) and ReadiDropTM propidium iodide (PI, 1351101, Bio-Rad) for $1 \mathrm{~h}$ at $4^{\circ} \mathrm{C}$ protect from light. Apoptotic cells were detected with Beckman Coulter Gallios Flow Cytometer and analyzed using FlowJo software (FlowJo, Ashland, USA).

\section{Wound healing assay}

ZR-75-30 cells were digested with trypsin and transferred to a 6-well plate and cultured for $24 \mathrm{~h}$. The wound was cut open with a $10 \mu \mathrm{L}$ pipette tip, and then washed off the floating cells with PBS. And take pictures to calculate the initial area. The culture was continued for $48 \mathrm{~h}$, observed under a microscope at the corresponding time points, and photographed.

\section{Transwell assay}

ZR-75-30 cells $\left(2 \times 10^{5} / \mathrm{mL}\right)$ at logarithmic growth stage were suspended in $200 \mu \mathrm{L}$ serum-free RPMI 1640 medium, and the cell suspension was added into the upper chamber of Transwell with matrix glue and the lower chamber with $500 \mu \mathrm{L}$ FBS. After cultured for $24 \mathrm{~h}$ and the cells that did not pass through the stomata were plucked out with swabs. The cells were immovable with $4 \%$ paraformaldehyde and pigmented with Giemsa. Five visual points were haphazardly picked under the microscope to take photos and counting. The average number of cells penetrating to the lower chamber was taken as the experimental results.

\section{Quantitative real-time PCR (qPCR)}

RNA was extracted from ZR-75-30 cells according to the instructions of RNA extraction kit (Invitrogen, USA). After synthesizing the first strand of CDNA, the SYBR Green method was used for Real-time PCR, 
and $\beta$-actin was used as internal reference to detect the level of mRNA. There were 3 multiple pores in each sample, and the data were analyzed by $2^{-\Delta \Delta C t}$ method. The primer sequence are used as follows: NUPR1, 5'-AGG ACT TAT TCC CGC TGA CTG A-3' (Forward) and 5'-TGC CGT GCG TGT CTA TTT ATT G-3' (Reverse); TFE3, 5'-CCG TGT TCG TGC TGT TGG A-3' (Forward) and 5'-GCT CGT AGA AGC TGT CAG GAT3' (Reverse); $\beta$-actin, 5'-CAG CCT CAA GAT CAT CAG CA-3' (Forward) and 5'-TGT GGT CAT GAG TCC TTC CA-3' (Reverse).

\section{Western blot}

ZR-75-30 cells were extracted the total protein, and the protein content was determined by BCA kits (Beyotime, Shanghai, China). The same protein content samples were boiled for $10 \mathrm{~min}$, and then subjected to SDS-PAGE, membrane transfer, sealed overnight, membrane washing, primary antibody incubation, membrane washing, secondary antibody incubation, and ECL chemiluminescence development. The results were analyzed by Tanon chemiluminescence imaging analysis system. The primary antibody information is shown below: TFE3 (1:500, ab196681), LC3 (1:1000, ab229327), p62 (1:1000, ab91526), Beclin1 (1:1000, ab62557), ATG5 (1:1000, ab228668), E-cadherin (E-cad, 1:1000, ab231303), N-cadherin (N-cad, 1:1000, ab207608), vimentin (1:1000, ab137321) and $\beta$-actin (1:500, ab115777).

\section{Chromatin immunoprecipitation (ChIP)}

Breast cancer cells were digested into $200 \sim 1000$ bp of soluble chromatin by ultrasonic lysis, and DNA/protein immunoprecipitation was carried out. ChIP antibody was added and incubated overnight under the condition of $4^{\circ} \mathrm{C}$ to obtain DNA/protein precipitation. The DNA was dissociated and purified for qPCR. The primer sequence was synthesized by Sangon Biotech Co., Ltd (Shanghai, China).

\section{In vivo experiment}

All animal experimentations were consummated in obedience to stipulations approved by Ethics Committee of The Affiliated Zhuzhou Hospital of Xiangya Medical College CSU. Balb/c female nude mice (6-8 weeks) were obtained from Beijing Experimental Animal Center. Mice were maintained in a $12 \mathrm{~h}$ light/dark, $20-25^{\circ} \mathrm{C}$ and $50-65$ humidity specific pathogen free animal facility with adequate food and water ad libitum and were acclimated for 1 week before tumor cell inoculation. ZR-75-30 cells with stably expressing sh-NUPR1 were suspended in phosphate buffer $\left(1 \times 10^{6} / \mathrm{mL}\right)$. Cell suspension was inoculated subcutaneously into the axilla of nude mice. The dimensions of tumors were measured once a week. On day 49 , mice were euthanized before they become moribund. Tumors were isolated for further analysis.

\section{Statistical analysis}

GraphPad Prism 8 software was used to analyze the data, and the measurement data were expressed as Mean \pm SD. Student's test and analysis of variance were used for statistical processing. All the data were repeated for at least 3 independent experiments. A value of $P<0.05$ is considered significant. 


\section{Results}

\section{NUPR1 is upregulated in breast cancer tissues and cells}

Breast cancer/paracancerous tissues (30 cases) were collected for exploring the role of NUPR1. IHC showed the strongly positive expression of NUPR1 in breast cancer tissues (Fig. 1A). Simultaneously, the expression of NUPR1 mRNA was ascertained by qPCR, and the data presented that expression of NUPR1 mRNA in breast cancer tissues was considerably more elevated than that in paracancerous tissues (Fig. 1B). Further, the expression of NUPR1 in the tissues of patients with lymph node metastasis was considerably higher than that of patients without lymph node metastasis (Fig. 1C). Breast cancer patients with high NUPR1 expression exhibited shorter overall survival times than patients with low NUPR1 expression (Fig. 1D). In addition, the expression of NUPR1 was also dramatically increased in breast cancer cells MCF-7, MDA-MB-231, ZR-75-30, and T47D, as compared with normal breast epithelial cells MCF-10A. The results showed that the expression of NUPR1 in breast cancer cells was different, but it was significantly higher than that in normal breast epithelial cells MCF-10A (Fig. 1E). Since the expression of NUPR1 in ZR-75-30 was the highest, this cell was selected for the experiment in subsequent studies.

\section{Effects of NUPR1 knockdown on proliferation, migration and apoptosis of breast cancer cells}

To investigate the role of NUPR1 in breast cancer cells, we knocked down NUPR1 in ZR-75-30 cells. The knockdown efficiency of NUPR1 was ascertained by qPCR and the expression of NUPR1 in ZR-75-30 cells decreased significantly (Fig. 2A). The cell viability of ZR-75-30 cells with NUPR1 knockdown was notably inhibited at $48 \mathrm{~h}$ and $72 \mathrm{~h}$ (Fig. 2B). Cell proliferative activity of sh-NUPR1 group was remarkably attenuated (Fig. 2C). Similarly, the apoptosis rate of ZR-75-30 cells with sh-NUPR1 was remarkably increased (Fig. 2D). In the subsequent wound healing assay, we found that the migration ability of ZR-7530 cells with NUPR1 knockdown to the center after $48 \mathrm{~h}$ of incubation was remarkably weaker than that of sh-NC group, indicating that sh-NUPR1 had an inhibitory effect on cell migration (Fig. 2E). Simultaneously, the results of transwell assay showed that the invasive ability of cells was significantly inhibited in ZR-75-30 cells with sh-NUPR1 (Fig. 2F). The above results suggest that sh-NUPR1 can suppress the proliferation and migration of breast cancer cells and promote cell apoptosis.

\section{NUPR1-mediated autophagy promotes breast cancer metastasis}

To explore whether NURP1 affects the level of autophagy, western blot analysis was used to detect the expression levels of autophagy-related proteins in ZR-75-30 cells transfected with sh-NUPR1. The expression levels of LC3-II/LC3-I, Beclin1, ATG5 significantly decreased, and p62 dramatically increased after NUPR1 knockdown (Fig. 3A). These results indicated that NUPR1 knockdown could inhibit the activation of autophagy. E-cad, $\mathrm{N}$-cad, and vimentin were a participant in tumor metastasis and invasion. After NUPR1 knockdown in ZR-75-30 cells, the expression of E-cad was remarkably enhanced, whereas the expression of $\mathrm{N}$-cad and vimentin were significantly diminished, suggesting that NUPR1 knockdown could inhibit the metastasis of breast cancer (Fig. 3B). ATG5 is defined as an activator of autophagy, of 
which overexpression is considered to be a risk factor for recurrence in patients with breast cancer(22). The expression of E-cad was upregulated, while the expression of $\mathrm{N}$-cad and vimentin was significantly decreased in ZR-75-30 cells with ATG5 knockdown, indicating that inhibition of autophagy could inhibit breast cancer metastasis (Fig. $3 \mathrm{C}$ ). Interestingly, in wound healing assay, the migration ability of cells was significantly inhibited after ATG5 knockdown (Fig. 3D). Contemporaneously, the invasive ability of cells was significantly inhibited after ATG5 knockdown (Fig. 3E). The above results suggest that NUPR1 mediated autophagy to promote the metastasis of breast cancer.

\section{NUPR1 activates TFE3 transcription to promote autophagy}

TFE3 is considered to play an important role in autophagy. After NUPR1 knockdown, the expression of TFE3 mRNA and TFE3 protein were ascertained by qPCR and western blot, respectively. The results showed that the expression level of TFE3 mRNA decreased significantly (Fig. 4A), and the expression level of TFE3 protein also decreased significantly (Fig. 4B). In addition, according to the results of ChIP, it is not difficult to find that NUPR1 binds closely to the transcriptional region of TFE3 gene (Fig. 4C and D). This means that NUPR1 interacts directly with TFE3. Moreover, the data of luciferase reporter assay also implied that NUPR1 knockdown significantly decreased the transcriptional activity of TFE3 (Fig. 4E). Further, the expression levels of LC3-II/LC3-I, Beclin1, and ATG5 after TFE3 knockdown were significantly decreased, while p62 was notably upregulated, indicating that TFE3 knockdown inhibits the autophagy process (Fig. 4F). These results suggest that NUPR1 can activate TFE3 transcription and promote autophagy in breast cancer cells.

\section{NUPR1 knockdown inhibits tumor formation and metastasis in vivo}

To investigate the influence of NUPR1 on tumor metastasis and growth in vivo, the size of solid tumor in mice was measured and photographed. The size of solid tumor with sh-NUPR1 was remarkably smaller than that with sh-NC, indicating that NUPR1 knockdown remarkably obstruct the augmentation of tumor in vivo (Fig. 5A and B). By measuring the tumor weight, it was found that the tumor weight was significantly reduced after NUPR1 knockdown (Fig. 5C). According to the results of IHC, the expression of Ki67 in solid tumors of sh-NUPR1 group was significantly inhibited (Fig. 5D). In addition, compared with sh-NC group, it was not difficult to observe that the morphology of lung organs in sh-NUPR1 group was more normal, and NUPR1 knockdown significantly inhibited lung metastasis (Fig. 5E). The results of qPCR indicated that the expression of NURP1 and TFE3 in sh-NUPR1 group was remarkably decreased (Fig. 5F). The results of western blot showed that the autophagy-related proteins LC3-II/LC3-I, Beclin1, and ATG5 were significantly decreased, and p62 was increased in sh-NUPR1 group (Fig. 5G). Also, the expression of E-cad related to tumor invasion and metastasis increased and the expression of $\mathrm{N}$-cad and vimentin decreased within NUPR1 knockdown (Fig. 5H). The above results suggest that depletion of NUPR1 can inhibit tumor formation and metastasis in vivo.

\section{Discussion}


Breast cancer, a malignant tumor originating from breast tissue, is the most common cause of cancer death among women all over the world(23). Invasion and metastasis is an important biological behavior of tumor cells, which is tightly concerned with deficient prognosis and critically threatens the healthiness and lifespan of invalids with metastasis(24). Accordingly, in-depth analysis of the mechanism of breast cancer metastasis is of great significance to aggrandize the subsistence rate and prognosis of invalids with breast cancer. As claimed by researches, approximately $15 \%$ of breast cancer patients have metastases at the time of diagnosis, and $90 \%$ of breast cancer-related deaths are caused by metastasis $(25,26)$. Differential gene expression is a pivotal process in the development and metastasis of breast cancer. According to our research, abnormal expression of NUPR1 was found in cancerous tissues of 30 patients with breast cancer, while the overexpression of NUPR1 in 11 samples with lymph node metastasis was higher than that without lymph node metastasis.

Due to the uncontrolled growth of tumor cells, abnormal mitochondrial function and protein wrong folding, tumor cells survive under environmental and metabolic pressure for a long time. Autophagy becomes an important way for tumor cells to maintain the stability of their internal environment. Tumor cells can rely on the metabolic substrates produced by autophagy to maintain their own proliferation(27). Autophagy is regulated by a variety of genes and proteins. LC3 is a marker protein for detecting autophagy activity. A research based on 44 breast cancer patients with metastasis and 52 breast cancer patients with metastasis confirmed that the overexpression of autophagy-associated proteins LC3 and ATG17 in breast cancer was significantly correlated with the high metastasis rate(9). Beclin1 is a key regulator of autophagy signaling pathway. In the early stage of autophagy, Beclin 1 controls the formation of autophagosomes mainly by regulating the localization of other autophagy proteins to the membrane of pre-autophagy corpuscles(13). Studies have confirmed that Beclin1 is abnormally expressed in breast cancer tissues with positive lymph node metastasis, positive distal metastasis and positive-Ki67(28). ATG5, an important gene involved in the regulation of autophagy, can bind with ATG12 to form a complex located on the surface of autophagosome membrane, which can induce the formation of autophagosome by promoting the extension and expansion of autophagosome membrane(14). We found that after ATG5 knockdown, the expression of E-cad related to tumor invasion and metastasis was enhanced, whereas the expression of $\mathrm{N}$-cad and vimentin were diminished, suggesting that inhibition of autophagy could inhibit the metastasis of breast cancer. Moreover, transwell assay and wound healing assay confirmed that the invasion and migration ability were remarkably inhibited after ATG5 knockdown.

NUPR1 can provoke human breast cancer, and it is concerned with the metastasis and poor prognosis of breast cancer, suggesting that NUPR1 may be a key gene in promoting distant metastasis and growth of tumor cells(29). According to Hamidi, et al., overexpression of NUPR1 could hinder the transcription and expression of related genes under hypoxia and glucose starvation stress. Cell death induced by hypoxia and glucose starvation is autophagic death(30). Interestingly, in the study of non-small cell lung cancer, NUPR1 was found to initiate the transcription of genes related to autophagy-lysosomal pathway, thus initiating the expression of a series of genes, including autophagosome membrane formation, the genesis and maturation of autophagosome, the fusion of autophagosome and lysosome, lysosome 
generation and so on $(13,16)$. Zeng, et al., studied the efficacity of down-regulation of NUPR1 gene on proliferation and apoptosis of human multiple myeloma U266 cells by NUPR1 knockdown. It was found that the proliferation of U266 cells were decreased and the apoptosis rate was increased significantly after silencing NUPR1(31). These results confirm the effect of NUPR1 on tumor cells, but evidence is lacking on whether and how NUPR1 plays a role in breast cancer. In this research, ChIP suggested that NUPR1 interacts directly with TFE3. NUPR1 knockdown was found to decrease the expression of TFE3 mRNA and TFE3 protein. Furthermore, NUPR1 knockdown showed that the proliferation, migration and invasion of breast cancer cells were suppressed, and the apoptosis of ZR-75-30 cells was increased. In accordance with the results of western blot, knockdown of NUPR1 or TFE3 can inhibit autophagy and metastasis of breast cancer cells. In vivo experiments, it was found that NUPR1 knockdown can inhibit tumor formation and metastasis.

\section{Conclusions}

In summary, we clarified the significance of NUPR1 in breast cancer metastasis and confirmed the molecular mechanism of NUPR1 promoting breast cancer metastasis by activating autophagy through the TFE3 signaling pathway, providing a biological basis for intervention to block distant metastasis.

\section{Abbreviations}

NUPR1, Nuclear protein 1; TFE3, Translocation factor E3; DMEM, Dulbecco's modified eagle medium; FBS, Fetal bovine serum; CCK-8, Cell counting kit-8; sh, shRNA; PI, Propidium; PBS, Phosphate buffered saline; ChIP, Chromatin immunoprecipitation; qPCR, Quantitative real-time PCR; E-cad, E-cadherin; N-cad, Ncadherin.

\section{Declarations}

\section{Acknowledgements}

We would like to give our sincere gratitude to the reviewers for their constructive comments.

\section{Funding}

None.

\section{Ethics Approval and Consent to Participate}

All human participants provided written informed consent to participate in this research, which was approved by Ethics Committee of The Affiliated Zhuzhou Hospital of Xiangya Medical College CSU. All animal experimentations were consummated in obedience to stipulations approved by Ethics Committee of The Affiliated Zhuzhou Hospital of Xiangya Medical College CSU. 


\section{Consent for Publication}

The informed consent was obtained from study participants.

\section{Availability of Data and Material}

All data generated or analyzed during this study are included in this article. The datasets used and/or analyzed during the current study are available from the corresponding author on reasonable request.

\section{Competing Interests}

The authors declare that there is no conflict of interest.

\section{Authors' Contributions}

HX: Conceptualization; Writing-original draft; Methodology; Formal analysis;

JL: Supervision; Validation; Data curation;

XC: Investigation; Software; Visualization;

MDT: Funding acquisition; Project administration; Resources; Writing-review \& editing.

All authors have read and approved the final version of this manuscript to be published.

\section{References}

1. Bray F, Ferlay J, Soerjomataram I, Siegel RL, Torre LA, Jemal A. Global cancer statistics 2018 : GLOBOCAN estimates of incidence and mortality worldwide for 36 cancers in 185 countries. CA: a cancer journal for clinicians. 2018;68(6):394-424.

2. Jansana A, Posso M, Guerrero I, Prados-Torres A, Del Cura MI, Castells X, et al. Health care services use among long-term breast cancer survivors: a systematic review. Journal of cancer survivorship : research and practice. 2019;13(3):477-93.

3. Yang SS, Ma S, Dou H, Liu F, Zhang SY, Jiang C, et al. Breast cancer-derived exosomes regulate cell invasion and metastasis in breast cancer via miR-146a to activate cancer associated fibroblasts in tumor microenvironment. Experimental cell research. 2020;391(2):111983.

4. Goodwin JM, Dowdle WE, DeJesus R, Wang Z, Bergman P, Kobylarz M, et al. Autophagy-Independent Lysosomal Targeting Regulated by ULK1/2-FIP200 and ATG9. Cell reports. 2017;20(10):2341-56.

5. Nikoletopoulou V, Markaki M, Palikaras K, Tavernarakis N. Crosstalk between apoptosis, necrosis and autophagy. Biochimica et biophysica acta. 2013;1833(12):3448-59.

6. Mowers EE, Sharifi MN, Macleod KF. Autophagy in cancer metastasis. Oncogene. 2017;36(12):161930 . 
7. Su Z, Yang Z, Xu Y, Chen Y, Yu Q. Apoptosis, autophagy, necroptosis, and cancer metastasis. Molecular cancer. 2015;14:48.

8. Gu Y, Li P, Peng F, Zhang M, Zhang Y, Liang H, et al. Autophagy-related prognostic signature for breast cancer. Molecular carcinogenesis. 2016;55(3):292-9.

9. Hashemi-Sadraei N, Muller-Greven GM, Abdul-Karim FW, Ulasov I, Downs-Kelly E, Burgett ME, et al. Expression of LC3B and FIP200/Atg17 in brain metastases of breast cancer. Journal of neurooncology. 2018;140(2):237-48.

10. Qin Z, Xue J, He Y, Ma H, Jin G, Chen J, et al. Potentially functional polymorphisms in ATG10 are associated with risk of breast cancer in a Chinese population. Gene. 2013;527(2):491-5.

11. Wu CL, Zhang SM, Lin L, Gao SS, Fu KF, Liu XD, et al. BECN1-knockout impairs tumor growth, migration and invasion by suppressing the cell cycle and partially suppressing the epithelialmesenchymal transition of human triple-negative breast cancer cells. International journal of oncology. 2018;53(3):1301-12.

12. Tan M, Wu A, Liao N, Liu M, Guo Q, Yi J, et al. Inhibiting ROS-TFE3-dependent autophagy enhances the therapeutic response to metformin in breast cancer. Free radical research. 2018;52(8):872-86.

13. Mu Y, Yan X, Li D, Zhao D, Wang L, Wang X, et al. NUPR1 maintains autolysosomal efflux by activating SNAP25 transcription in cancer cells. Autophagy. 2018;14(4):654-70.

14. Galichon P, Bataille A, Vandermeersch $S$, Wetzstein $M, X u$-Dubois $Y C$, Legouis $D$, et al. Stress Response Gene Nupr1 Alleviates Cyclosporin A Nephrotoxicity In Vivo. Journal of the American Society of Nephrology : JASN. 2017;28(2):545-56.

15. Lee YK, Jee BA, Kwon SM, Yoon YS, Xu WG, Wang HJ, et al. Identification of a mitochondrial defect gene signature reveals NUPR1 as a key regulator of liver cancer progression. Hepatology. 2015;62(4):1174-89.

16. Schroll MM, LaBonia GJ, Ludwig KR, Hummon AB. Glucose Restriction Combined with Autophagy Inhibition and Chemotherapy in HCT 116 Spheroids Decreases Cell Clonogenicity and Viability Regulated by Tumor Suppressor Genes. Journal of proteome research. 2017;16(8):3009-18.

17. Jung SH, Lee A, Yim SH, Hu HJ, Choe C, Chung YJ. Simultaneous copy number gains of NUPR1 and ERBB2 predicting poor prognosis in early-stage breast cancer. BMC cancer. 2012;12:382.

18. Jiang WG, Watkins G, Douglas-Jones A, Mokbel K, Mansel RE, Fodstad O. Expression of Com-1/P8 in human breast cancer and its relevance to clinical outcome and ER status. International journal of cancer. 2005;117(5):730-7.

19. Ito Y, Yoshida H, Motoo Y, lovanna JL, Nakamura Y, Kakudo K, et al. Expression of p8 protein in breast carcinoma; an inverse relationship with apoptosis. Anticancer research. 2005;25(2A):833-7.

20. Fan T, Pi H, Li M, Ren Z, He Z, Zhu F, et al. Inhibiting MT2-TFE3-dependent autophagy enhances melatonin-induced apoptosis in tongue squamous cell carcinoma. Journal of pineal research. 2018;64(2).

21. Perera RM, Stoykova S, Nicolay BN, Ross KN, Fitamant J, Boukhali M, et al. Transcriptional control of autophagy-lysosome function drives pancreatic cancer metabolism. Nature. 2015;524(7565):361-5. 
22. Wible DJ, Chao HP, Tang DG, Bratton SB. ATG5 cancer mutations and alternative mRNA splicing reveal a conjugation switch that regulates ATG12-ATG5-ATG16L1 complex assembly and autophagy. Cell discovery. 2019;5:42.

23. Yang C, Tabatabaei SN, Ruan X, Hardy P. The Dual Regulatory Role of MiR-181a in Breast Cancer. Cellular physiology and biochemistry : international journal of experimental cellular physiology, biochemistry, and pharmacology. 2017;44(3):843-56.

24. Hur K, Toiyama Y, Okugawa Y, Ide S, Imaoka H, Boland CR, et al. Circulating microRNA-203 predicts prognosis and metastasis in human colorectal cancer. Gut. 2017;66(4):654-65.

25. Metzger-Filho O, Tutt A, de Azambuja E, Saini KS, Viale G, Loi S, et al. Dissecting the heterogeneity of triple-negative breast cancer. Journal of clinical oncology : official journal of the American Society of Clinical Oncology. 2012;30(15):1879-87.

26. Foroni C, Broggini M, Generali D, Damia G. Epithelial-mesenchymal transition and breast cancer: role, molecular mechanisms and clinical impact. Cancer treatment reviews. 2012;38(6):689-97.

27. Pengo N, Scolari M, Oliva L, Milan E, Mainoldi F, Raimondi A, et al. Plasma cells require autophagy for sustainable immunoglobulin production. Nature immunology. 2013;14(3):298-305.

28. Rohatgi RA, Janusis J, Leonard D, Bellve KD, Fogarty KE, Baehrecke EH, et al. Beclin 1 regulates growth factor receptor signaling in breast cancer. Oncogene. 2015;34(42):5352-62.

29. Ree AH, Tvermyr M, Engebraaten O, Rooman M, Rosok O, Hovig E, et al. Expression of a novel factor in human breast cancer cells with metastatic potential. Cancer research. 1999;59(18):4675-80.

30. Hamidi T, Cano CE, Grasso D, Garcia MN, Sandi MJ, Calvo EL, et al. Nupr1-aurora kinase A pathway provides protection against metabolic stress-mediated autophagic-associated cell death. Clinical cancer research : an official journal of the American Association for Cancer Research. 2012;18(19):5234-46.

31. Zeng C, Li X, Li A, Yi B, Peng X, Huang X, et al. Knockdown of NUPR1 inhibits the growth of U266 and RPMI8226 multiple myeloma cell lines via activating PTEN and caspase activationdependent apoptosis. Oncology reports. 2018;40(3):1487-94.

\section{Figures}


A

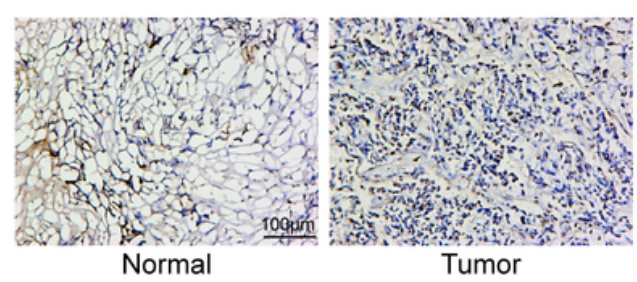

Normal

Tumor

D

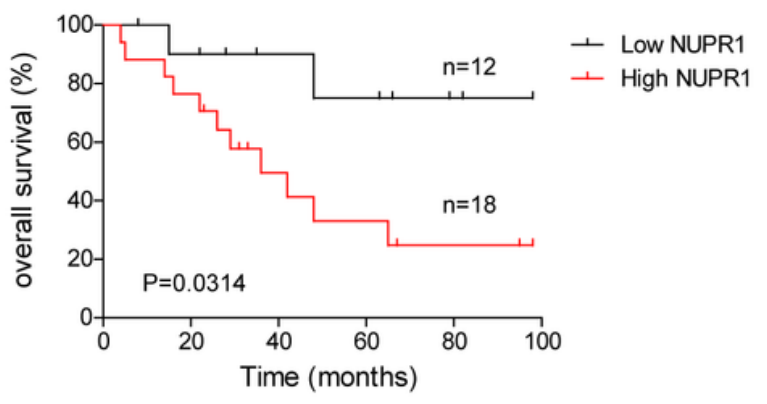

B

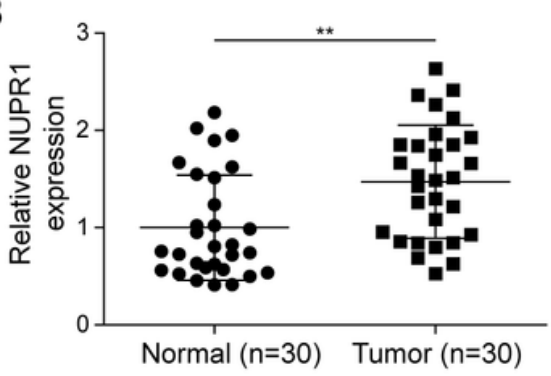

$\mathrm{E}$
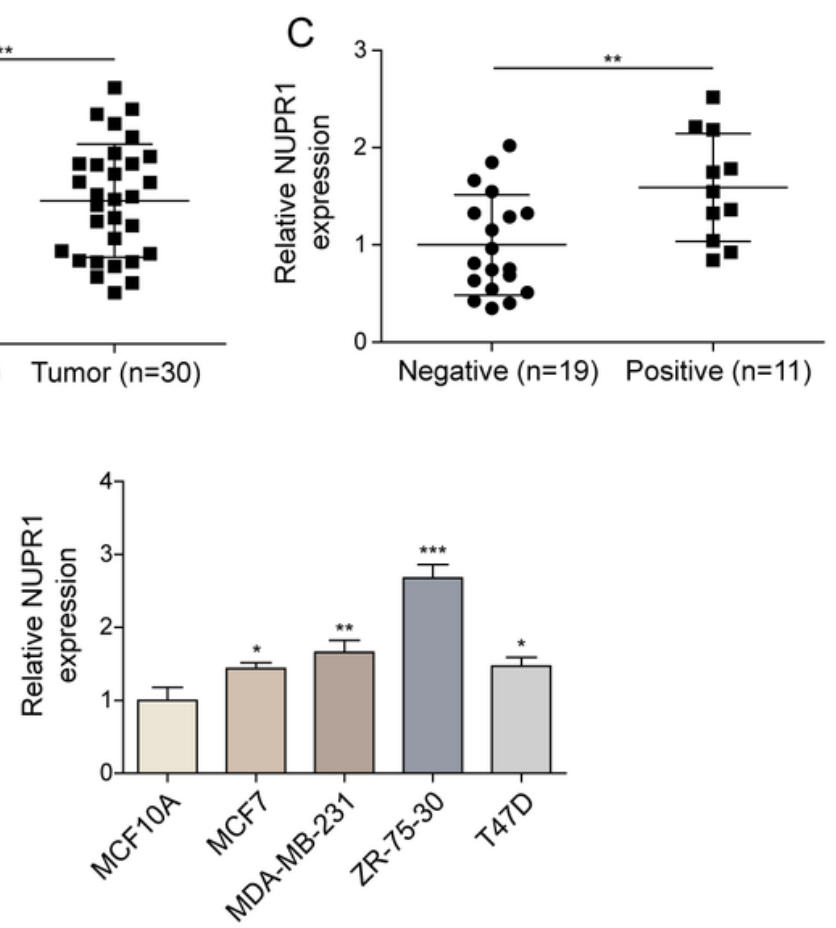

\section{Figure 1}

NUPR1 is upregulated in breast cancer tissues and cells (A) NUPR1 expression in breast cancer tissues was ascertained by IHC. (B) NUPR1 mRNA expression was detected by qPCR in breast cancer tissues. (C) NUPR1 expression in the tissues of patients with lymph node metastasis was ascertained by qPCR. (D) Kaplan-Meier survival of breast cancer patients with high and low NUPR1 expression, and the cut-off value was the median of NUPR1 value. (E) qPCR was used to reveal NUPR1 expression in breast cancer cells and normal breast epithelial cells. ${ }^{*} P<0.05,{ }^{*} P<<0.01$, and ${ }^{\star * \star} P<0.001$. 
A

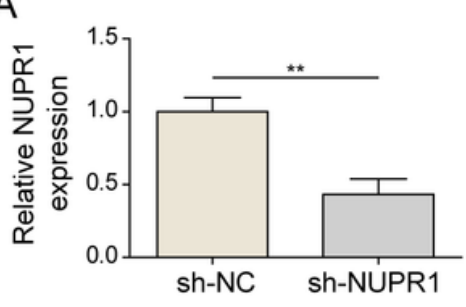

B

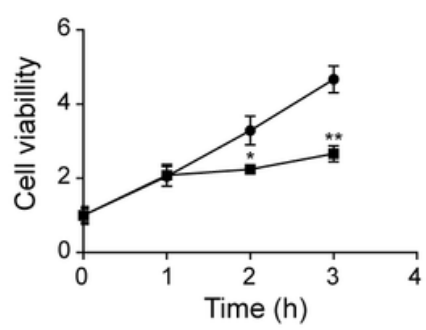

C

- sh-NC
- sh-NUPR1
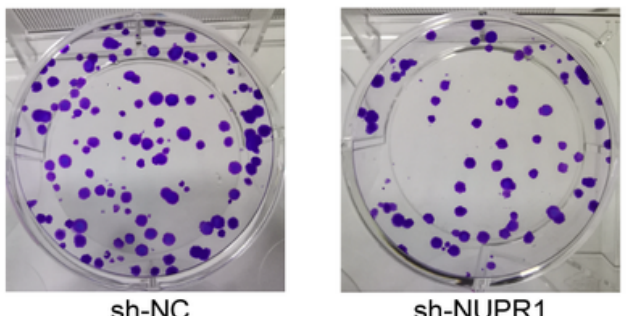

sh-NUPR1
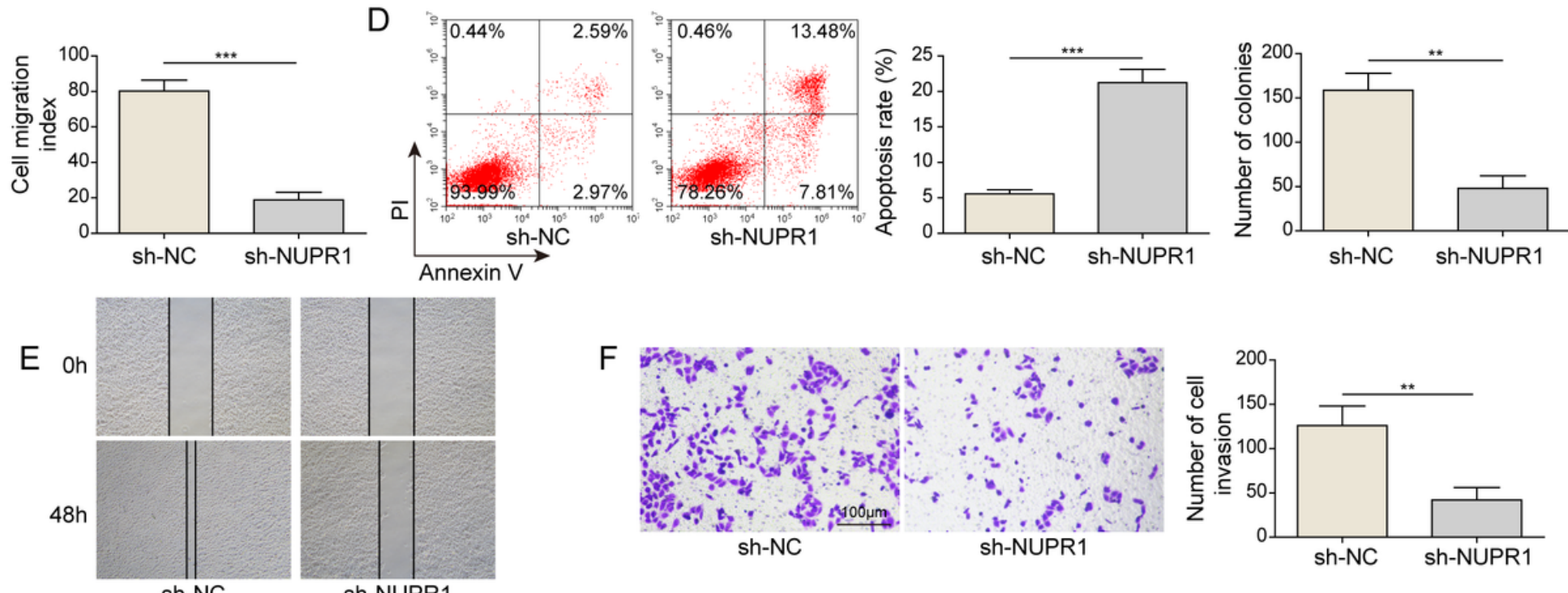

$\mathrm{F}$
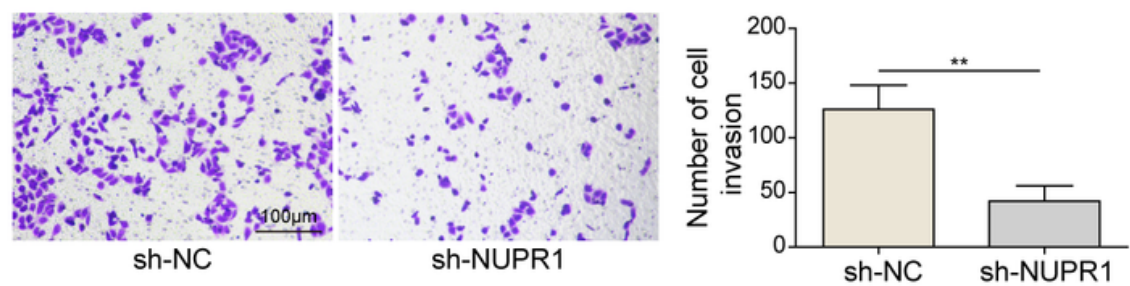

Figure 2

Effects of NUPR1 knockdown on proliferation, migration and apoptosis of breast cancer cells (A) Knockdown efficiency of NUPR1 was ascertained by qPCR. (B) Cell viability of ZR-75-30 cells with NUPR1 knockdown was ascertained by CCK-8 assay. (C) The cell proliferation of sh-NUPR1 group was detected by colony formation assay. (D) Apoptosis rate of ZR-75-30 cells with sh-NUPR1 was detected by flow cytometry. (E) Wound healing assay was detected migration ability of ZR-75-30 cells with NUPR1 knockdown. (F) Transwell assay was detected invasive ability of ZR-75-30 cells with sh-NUPR1. ${ }^{*} P<0.05$, $\star \star P<0.01$, and $* \star * P<0.001$. 


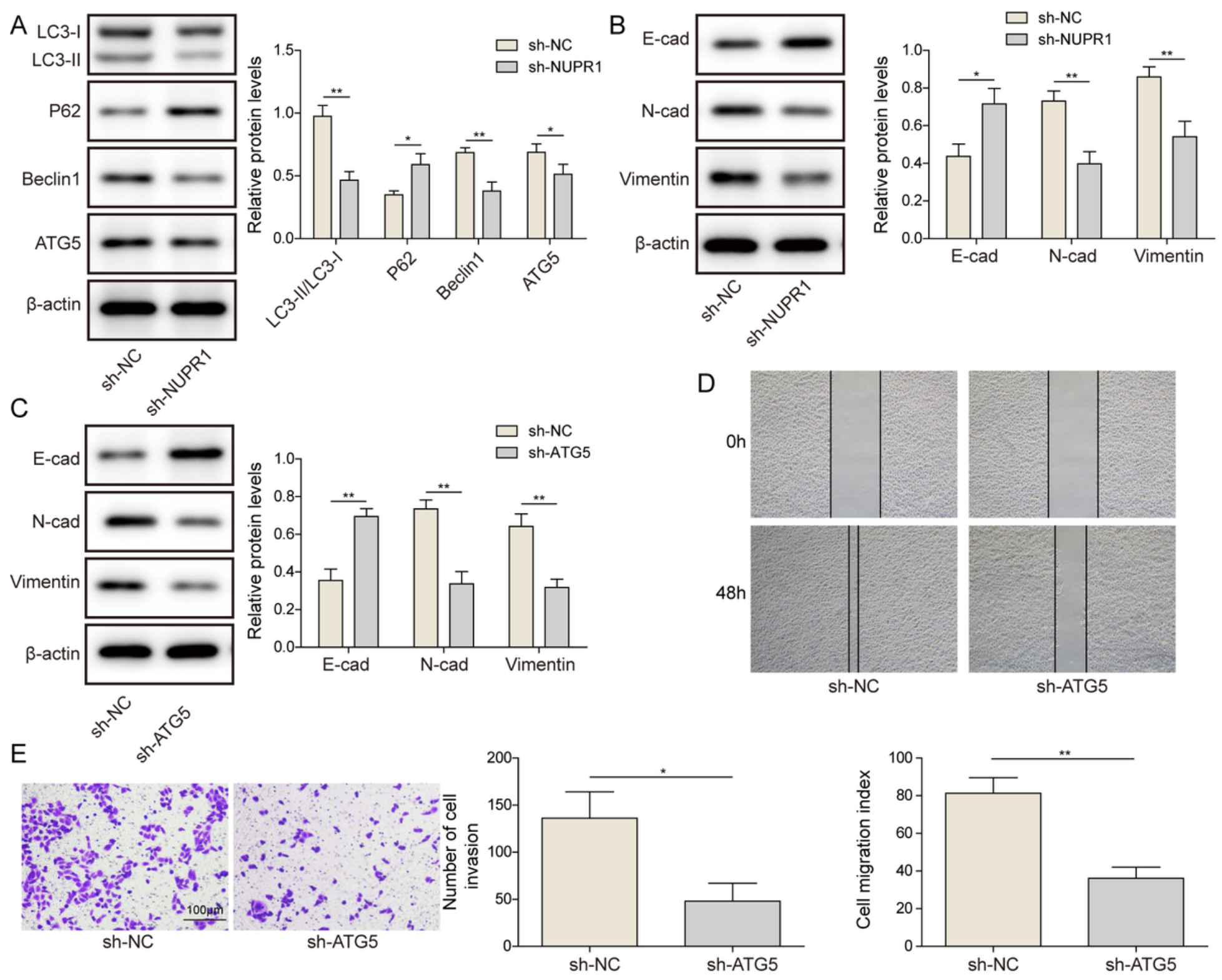

Figure 3

NUPR1-mediated autophagy promotes breast cancer metastasis. (A) The expression levels of autophagyrelated proteins in ZR-75-30 cells transfected with sh-NUPR1 were ascertained by western blot. (B) The expression levels of E-cad, N-cad, and vimentin in ZR-75-30 cells transfected with sh-NUPR1 were ascertained by western blot. (C) The expression levels of E-cad, N-cad, and vimentin in ZR-75-30 cells transfected with sh-ATG5 were ascertained by western blot. (D) Wound healing assay was detected migration ability of ZR-75-30 cells with ATG5 knockdown. (E) Transwell assay was detected invasive ability of ZR-75-30 cells transfected with sh-ATG5. ${ }^{*} P<0.05, * * P<0.01$. 

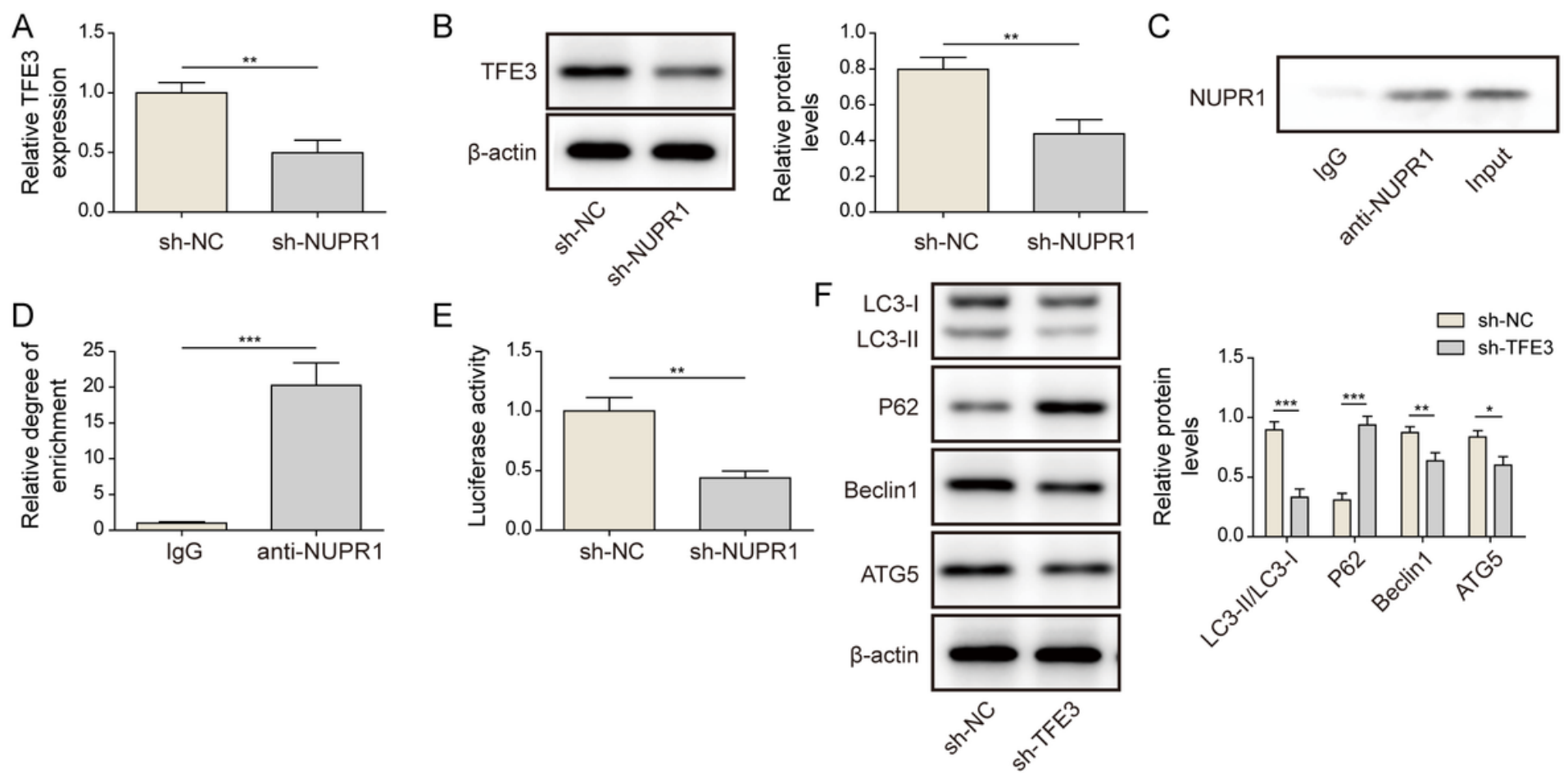

\section{Figure 4}

NUPR1 activates TFE3 transcription to promote autophagy. (A) The TFE3 mRNA expression in ZR-75-30 cells transfected with sh-NUPR1 was detected by qPCR. (B) The TFE3 protein expression in ZR-75-30 cells transfected with sh-NUPR1 was detected by western blot. ( $C$ and D) NUPR1 binds to the transcriptional region of TFE3 gene was detected by ChIP. (E) Luciferase reporter assay was performed to evaluate the transcriptional activity of TFE3 within NUPR1 knockdown. (F) The expression levels of LC3-I/LC3-II, p62, Beclin1, and ATG5 in breast cancer cells with TFE3 knockdown were detected by western blot. ${ }^{*} \mathrm{P}<0.05$, $\star \star P<0.01$, and $* * * P<0.001$. 

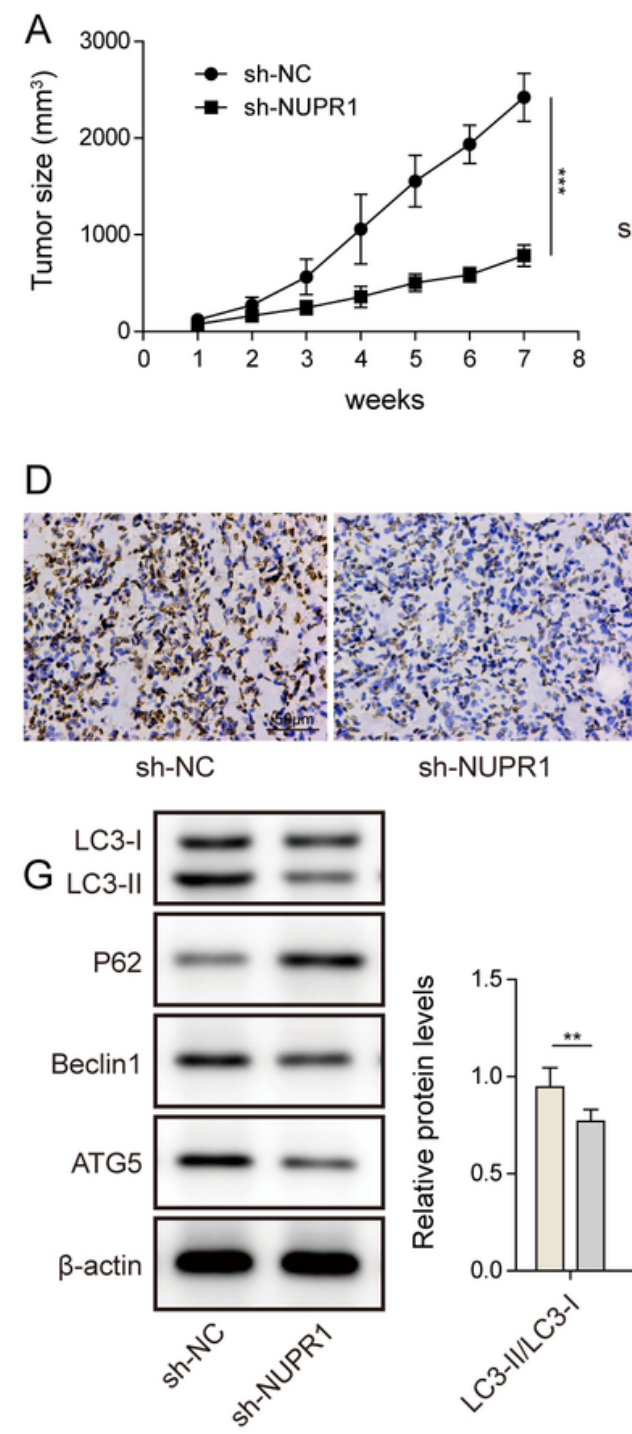
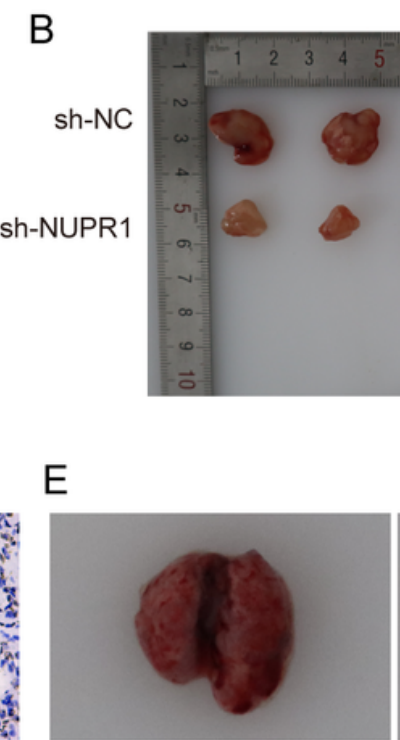

sh-NC
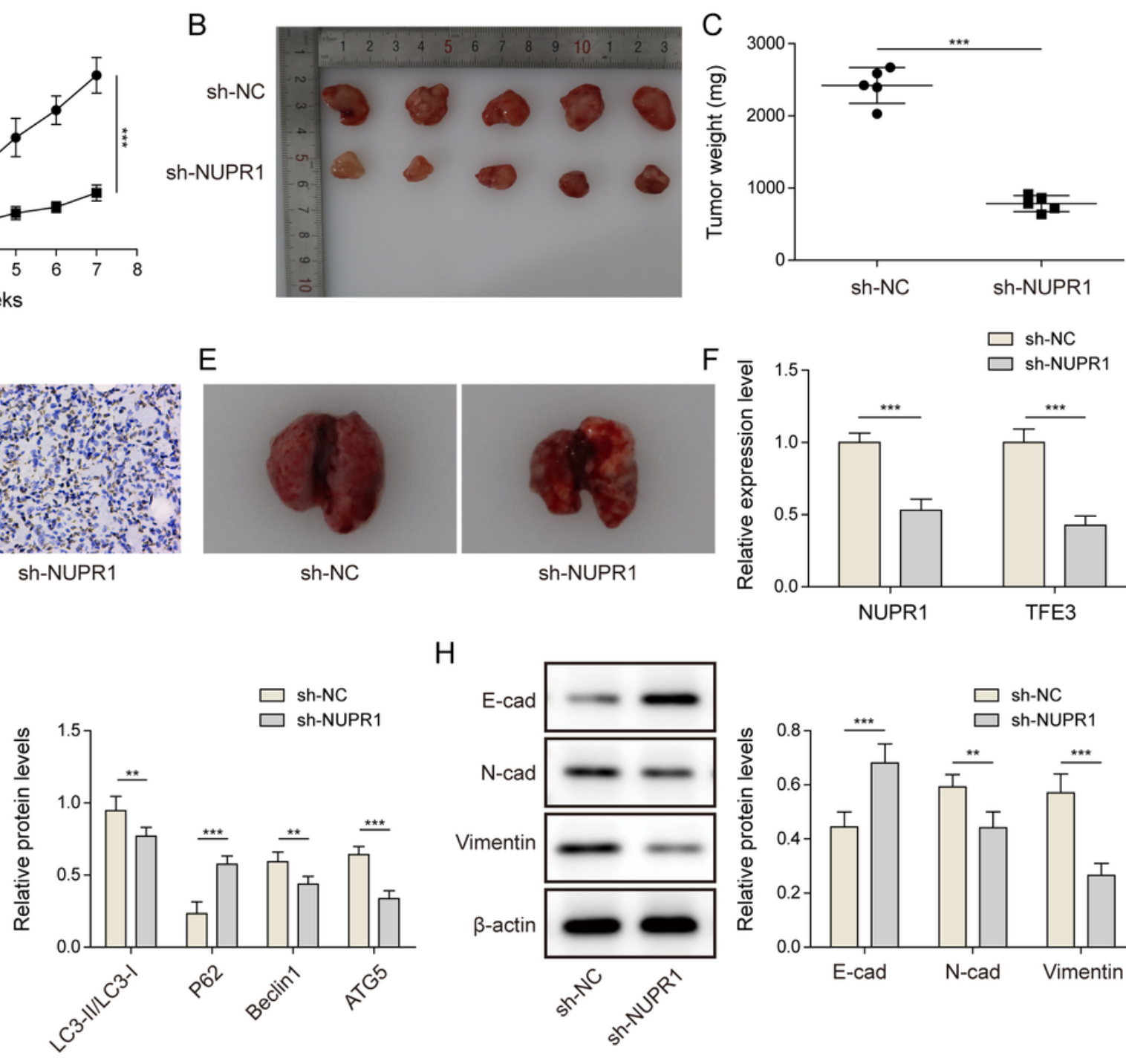

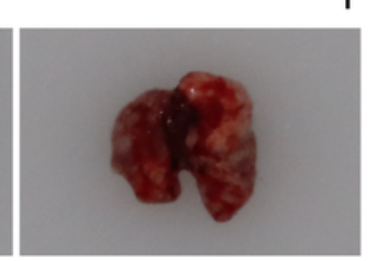

sh-NUPR1

$\mathrm{H}$

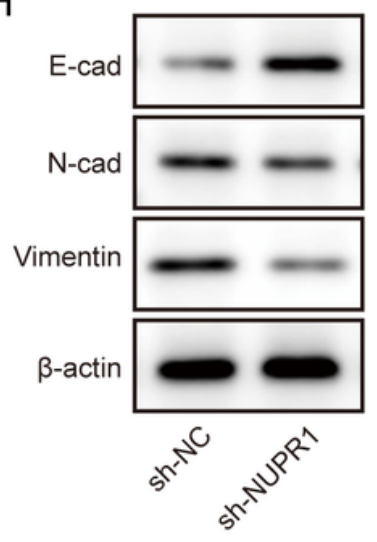

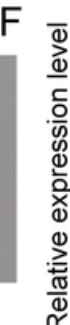
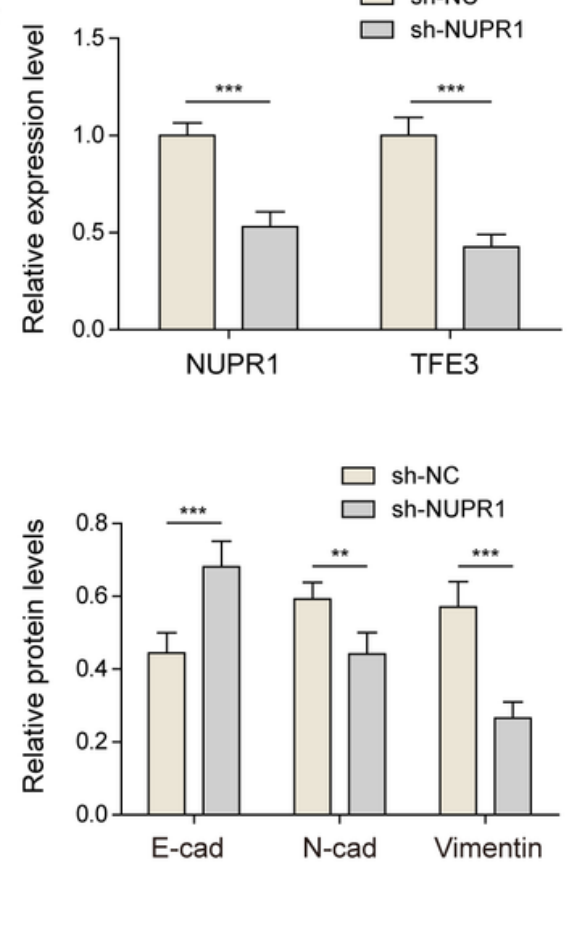

\section{Figure 5}

NUPR1 knockdown inhibits tumor formation and metastasis in vivo. (A and B) The size of solid tumor in mice was measured and photographed. (C) The tumor weight was measured after NUPR1 knockdown.

(D) Ki67 expression in solid tumors was detected by IHC. (E) The morphology of lung organs in sh-NUPR1 group was measured and photographed. (F) qPCR was detected to the expression of NURP1 and TFE3 in sh-NUPR1 group. (G) The expression levels of LC3-I/LC3-II, p62, Beclin1, and ATG5 were detected by western blot. $(\mathrm{H})$ The expression levels of E-cad, $\mathrm{N}$-cad, and vimentin were ascertained by western blot. $\star * P<0.01, * \star * P<0.001$. 